\title{
An Analytical Framework for Neighbor Discovery Strategies in Ad hoc Networks with Sectorized Antennas
}

\author{
Joon-Sang Park, Member, IEEE, Sung-woo Cho, M. Y. Sanadidi, Senior Member, IEEE, \\ and Mario Gerla, Fellow, IEEE,
}

\begin{abstract}
This letter analyzes a class of neighbor discovery algorithms in ad hoc networks using directional antennas for both reception and transmission. We consider synchronized and random sector - timeslot assignment strategies and compare their behaviors. Analytic results validated by simulation clearly show the latency benefits of the synchronized sector assignment over the random assignment.
\end{abstract}

Index Terms-Ad hoc Networks, Directional Antenna, Neighbor Discovery, Performance Analysis, and Poisson Distribution.

\section{INTRODUCTION}

In this letter, we develop a mathematical model to analyze a class of neighbor discovery strategies in ad hoc networks with directional-mode-only antennas (i.e., antennas that cannot operate in omni-directional mode) where transmit/receive pairs must align their transmission/reception directions. Neighbor discovery is a process of finding one-hop neighbors and is the first step towards the design of MAC, and routing protocols in ad hoc networks with nodes using directional antenna. The faster the nodes discover their neighbors, the higher the network availability and thus the performance.

Earlier works [1], [2], [6], [7], [9] in beamforming network setup relied on the use of omni-directional transmission/reception. [2], [7], [9] use omni-directional transmissions to advertise a node's location and ask listening nodes to send an acknowledgement as a response [7] and thus their works are limited by the range of these omni-directional advertisements and do not exploit the full range extension of the beamforming antennas. Neighbor discovery without omni-directional transmission/reception have been proposed in [3], [4], [5], [9]. [9] developed a probabilistic schedule of transmissions and receptions in randomly selected directions, while [4], [5] introduced a discovery strategy where all the nodes are synchronized in their transmit/receive direction selection but the decision to either transmit or listen is left at random. The protocol proposed in [4] assumes different beamwidths for transmission and reception and gradually increases transmission power in consideration of low probability of detection. [3] proposes

J.-S. Park is with the Department of Computer Engineering, Hongik University, Seoul 121-791, Korea. (email: jsp@ hongik.ac.kr)

S. Cho is with School of Business, Yonsei University, Seoul 120-749, Korea. (email: swcho@yonsei.ac.kr)

M. Sanadidi and M. Gerla are with the Computer Science Department University of California, Los Angeles, Los Angeles, CA 90095 USA (email: medy@cs.ucla.edu; gerla@cs.ucla.edu)
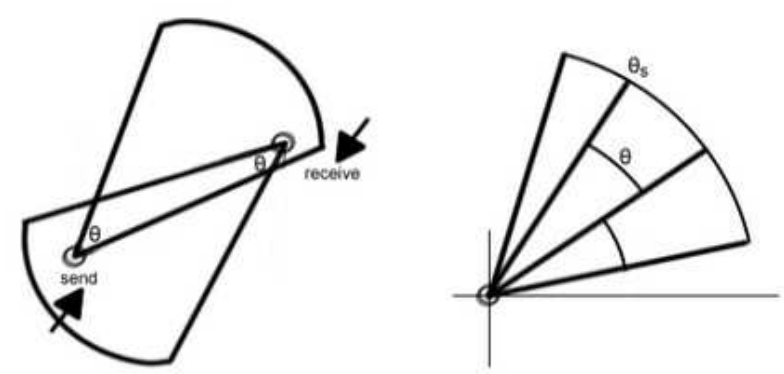

(a) Sender/Receiver both facing each (b) $\theta_{S}$ is the size of the supersector other within their sectors for a direc- and $\theta$ is the size of a sector, i.e., tional transmission. beamwidth.

Fig. 1. Directional transmission and comparison of supersector and sector

an integrated neighbor discovery and topology management mechanism for mobile nodes.

In this work, we analytically study and compare the randomized and synchronized approaches. We develop a neighbor discovery framework embracing these two approaches. We then perform numerical analysis to identify the best strategy in the class of neighbor discovery algorithms that can be represented by our framework. An analytical model for randomized neighbor discovery algorithms is presented in [9]; however, due to its inherent limitation the model can only be used to analyze randomized neighbor discovery algorithms and thus cannot be used to compare synchronized and randomized algorithms. The rest of this letter is organized as follows. In Section II, we describe our neighbor discovery analysis framework. Numerical analysis of discovery strategies is provided in Section III and Section IV concludes this letter.

\section{Problem Formulation}

Our neighbor discovery framework targets antenna systems that perform transmission/reception only in directional mode. A directional transmission can be received only by the nodes in a certain area; and similarly, a directional reception can receive signals transmitted from nodes only in a certain area. Those areas are approximated by circular sectors and the term beam is used to denote those sector shape areas. In our framework, we use an idealized channel model and ignore sidelobes for simplicity. Further, we assume that the transmission and reception beams are of the same size, called beamwidth. Since transmission and reception are both directional, the transmitter 
and receiver must be in the reception and transmission beam respectively (Fig. 1(a)) to make a successful data transfer.

The search space surrounding a node is divided into subspaces and time is divided into slots. In a given timeslot, each node either transmit with probability $p_{t}$ or listen with probability $\left(1-p_{t}\right)$ using half-duplex radio. Each timeslot has an associated subspace called supersector. Each of equalsized supersectors is composed of $m$ non-overlapping sectors the size of which is the same as beamwidth. (The supersector size determines $m$.) A node can randomly choose a sector within the given supersector for transmission or the one in the opposite side for reception. $\theta_{S}$ refer to the supersector size and $\theta$ refer to the sector size (Fig. 1(b)). The supersector that a node uses is determined by fixed scheduling and it is scheduled such that the total number of timeslots allocated are the same for all supersectors. (E.g., all the supersectors are visited in sequence. The cycle is repeated as many times as necessary.) The supersector schedule is the same for all nodes, though each node individually decides randomly which sector to use, and whether to transmit or receive in the chosen sector.

A node experiences a collision if multiple sources transmit toward the node in the same timeslot. We assume that time (slot boundaries) and the azimuth angle are synchronized across all the nodes and such time and azimuth synchronization among nodes can be achieved with a location service (e.g., GPS) and a compass installed on each node. We also assume that the node distribution follows a two-dimensional Poisson point process [8]. Thus, the probability of $n$ nodes appearing

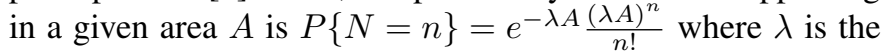
node density.

\section{ANALYSIS}

In this section, we develop a mathematical model to derive the latency required for neighbor discovery. We compare various search strategies from the fully synchronized search to the randomized search using the model. In the fully synchronized search, at each timeslot all the nodes are transmitting in a given direction or receiving in the opposite direction. All directions are explored in sequence. The randomized search assumes that in a given timeslot, nodes transmit or receive in a random direction. We model the fully synchronized search by setting the supersector size to the beamwidth and the randomized search by setting the supersector size to $2 \pi$.

Let $p_{i, j \mid K}$ be the probability that node $i$ discovers in a given timeslot a specific node $j$ located in a certain supersector $A$ given that there exist a total of $K$ nodes in $A$ including $j$. (Note that node $i$ 's position is excluded from A.) Node $i$ discovers node $j$, if node $i$ hears only $j$ 's transmission without any interference from other nodes in $i$ 's reception beam.

$$
p_{i, j \mid K}=\left(\frac{\theta_{S}}{2 \pi}\right)\left(\frac{\theta}{\theta_{S}}\right)^{2} p_{t}\left(1-p_{t}\right)\left(1-\left(\frac{\theta}{\theta_{S}}\right)^{2} p_{t}\right)^{K-1}
$$

The probability of $i$ 's exploration of $A$ in a given timeslot is $\frac{\theta_{S}}{2 \pi}$ (assuming that all supersectors are explored in a fair fashion), the probability of node $j$ 's transmission toward node $i$ is $\left(\frac{\theta}{\theta_{S}}\right) p_{t}$, the probability of node $i$ 's directing its reception towards node $j$ is $\left(\frac{\theta}{\theta_{S}}\right)\left(1-p_{t}\right)$, and the probability of all nodes other than node $j$ in node $i$ 's beam not transmitting toward node $i$ is $\left(1-\left(\frac{\theta}{\theta_{S}}\right)^{2} p_{t}\right)^{K-1}$ when there are $K$ nodes in the sector. $\frac{\theta}{\theta_{S}}$ is the probability of either $j$ or $i$ have their beams each encompassing the other and also the probability of a node other than $j$ being in $i$ 's beam assuming the two-dimensional Poisson node distribution.

Since any of $K$ nodes can be $j$ in (1), the probability of finding any node in a given timeslot given there exist $K$ nodes in the associated sector is

$p_{S \mid K}=K\left(\frac{\theta_{S}}{2 \pi}\right)\left(\frac{\theta}{\theta_{S}}\right)^{2} p_{t}\left(1-p_{t}\right)\left(1-\left(\frac{\theta}{\theta_{S}}\right)^{2} p_{t}\right)^{K-1}$.

Now we want to plot $E_{t}$ the expected fraction of neighbors discovered by a node within $t$ timeslots. To this end, we first derive $E_{t \mid K}$ as the expected fraction of neighbors discovered (number of nodes found within $t$ timeslots over total number of neighbors) by a node within $t$ timeslots, given that there are $K$ nodes in a sector, using the following recurrence equation:

$$
E_{t \mid K}-E_{t-1 \mid K}=\frac{1}{K} p_{S \mid K}\left(1-E_{t-1 \mid K}\right)
$$

That is, in the $t^{t h}$ timeslot, $E_{t \mid K}$ is increased by $\frac{1}{K}$, i.e., one newly discovered neighbor out of $\mathrm{K}$ neighbors, with probability $p_{S \mid K}\left(1-E_{t-1 \mid K}\right)$ since the node discovers any neighboring node with probability $p_{S \mid K}$ and the discovered node is new, i.e., the node has not been discovered in any previous timeslots, with probability $\left(1-E_{t-1 \mid K}\right)$. We solve (3) using z-transform and get

$$
E_{t \mid K}=1-\left(1-\frac{1}{K} p_{S \mid K}\right)^{t}=\sum_{j=1}^{t}\left(\begin{array}{l}
t \\
j
\end{array}\right)(-1)^{j-1}\left(\frac{p_{S \mid K}}{K}\right)^{j}
$$

Since $E_{t \mid 0}$ is undefined, i.e., no node is expected to be found if there is no node in neighborhood, we define $E_{t}$ as the expected faction of neighbors discovered by a node within $t$ timeslots given non-zero number of neighbors. That is,

$$
E_{t}=\frac{1}{P\{N>0\}} \sum_{n=1}^{\infty} P\{N=n\} E_{t \mid n}
$$

where $N$ denotes the random variable defined as the number of neighbors. And from (2), (4), (5), and the Poisson point process assumption, we get

$$
E_{t}=\frac{1}{1-e^{-\lambda A}} \sum_{n=1}^{\infty} e^{-\lambda A} \frac{(\lambda A)^{n}}{n !} E_{t \mid n}
$$

$$
=\frac{1}{1-e^{-\lambda A}} \sum_{n=1}^{\infty} e^{-\lambda A} \frac{(\lambda A)^{n}}{n !} \sum_{j=1}^{t}\left(\begin{array}{l}
t \\
j
\end{array}\right)(-1)^{j-1}\left(\frac{p_{S \mid n}}{n}\right)^{j}
$$




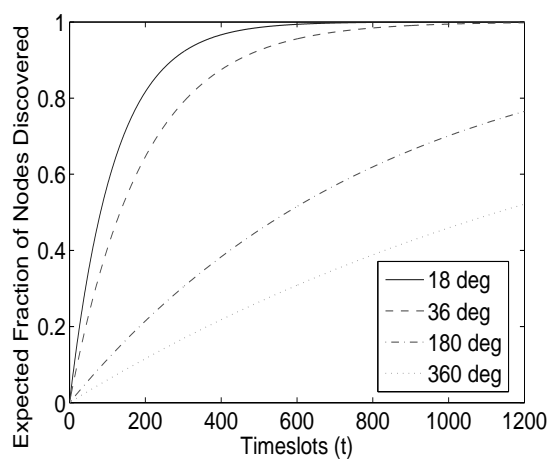

Fig. 2. Performance comparison using different supersector sizes with beamwidth of $0.1 \pi$

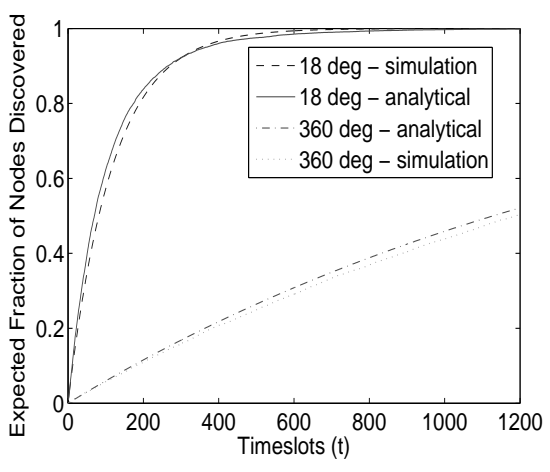

Fig. 3. Comparison of simulation and analytic results with beamwidth of $0.1 \pi$.

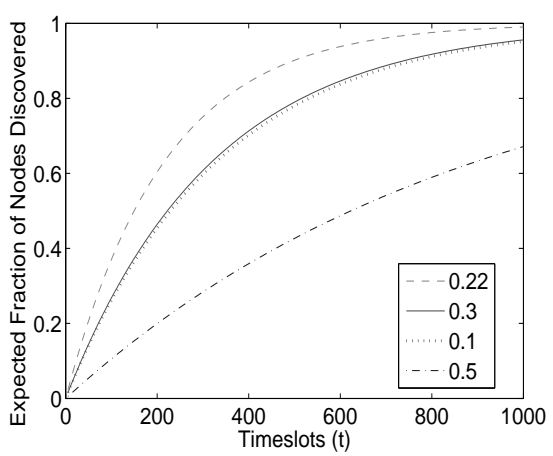

Fig. 4. Performance comparison using different $p_{t}$ 's.

$$
\begin{aligned}
& =\frac{1}{1-e^{-\lambda A}} \sum_{j=1}^{t}\left(\begin{array}{l}
t \\
j
\end{array}\right) \\
& \left(\left(\frac{\theta^{2} p_{t}\left(1-p_{t}\right)}{2 \pi \theta_{S}}\right)\left(1-\left(\frac{\theta}{\theta_{S}}\right)^{2} p_{t}\right)^{-1}\right)^{j} \\
& (-1)^{j-1} \sum_{n=1}^{\infty} e^{-\lambda A} \frac{(\lambda A)^{n}}{n !}\left(1-\left(\frac{\theta}{\theta_{S}}\right)^{2} p_{t}\right)^{j n} \\
& =\frac{1}{1-e^{-\lambda A}} \sum_{j=1}^{t}\left(\begin{array}{l}
t \\
j
\end{array}\right) \\
& \left(\left(\frac{\theta^{2} p_{t}\left(1-p_{t}\right)}{2 \pi \theta_{S}}\right)\left(1-\left(\frac{\theta}{\theta_{S}}\right)^{2} p_{t}\right)^{-1}\right)^{j} \\
& (-1)^{j-1}\left(e^{-\lambda A+\lambda A\left(1-\left(\frac{\theta}{\theta_{S}}\right)^{2} p_{t}\right)^{j}}-e^{-\lambda A}\right)
\end{aligned}
$$

where $A=\frac{\theta \pi r^{2}}{2 \pi}=\frac{\theta r^{2}}{2}$ is the reception beam of a node given the transmission/reception range $r$. Note that $P\{N>0\}=$ $(1-P\{N=0\})=\left(1-e^{-\lambda A}\right)$.

Fig. 2 plots $E_{t}$ for the beamwidth of $0.1 \pi$ as a function of $t$ for different supersector sizes with their optimal $p_{t}$ ' ${ }^{1}$. The figure shows that the best strategy is the fully synchronized search in which the supersector size is equal to beamwidth. The curve for the supersector size of $0.1 \pi$, the fully synchronized search, shows the steepest rise. Using the fully synchronized search, nodes are expected to find about $90 \%$ of their neighbors at $300^{\text {th }}$ timeslot whereas less than $20 \%$ using the randomized search. Setting the supersector size to $2 \pi$ is equivalent to having a random assignment strategy; the nodes can choose any direction to transmit and any direction to listen. It is also the worst strategy by far among all the various sizes chosen for the supersector. A node density of $\lambda=\frac{1000}{9 * 10^{6}}$ nodes $/ \mathrm{m}^{2}$ and a sector radius of $200 \mathrm{~m}$ is used in the figure.

Fig. 3 compares the analytical results to the simulation results obtained using a simple Monte Carlo simulation. Notice that simulation results are close to the analytic results. In the simulation, we use 1000 nodes randomly distributed over a $3000 \mathrm{~m}$ by $3000 \mathrm{~m}$ field and $200 \mathrm{~m}$ range to match parameters

\footnotetext{
${ }^{1}$ We solve the optimal $p_{t}$ by differentiating (5) and applying NewtonRalphson's method. The optimal $p_{t}$ when $\theta_{S}=\theta=0.1 \pi$ is approximately 0.38 .
}

used for the analytical results. The simulation results are averaged over 100 instances and the $95 \%$ confidence interval is $0.29 \pm 0.0027$ when $\mathrm{t}=600$ for the randomized search.

$p_{t}$ is an important parameter when optimizing systems' performance especially with high node densities. Some schemes (e.g., [4], [5]) set $p_{t}=0.5$, i.e., each node chooses to either transmit or receive at random; however, haphazardly using $p_{t}$ $=0.5$ results in a suboptimal performance as shown in Fig. 4 . We observe that less than $70 \%$ of neighbors are found with $p_{t}=0.5$ while near $100 \%$ neighbors are discovered with the optimal $p_{t}=0.22$ at $1000^{t h}$ timeslot. In the figure, we use $\lambda=\frac{5000}{9 * 10^{6}}$ nodes $/ m^{2}$ and $200 m$ range.

\section{CONCLUSION}

In this letter, we proposed a mathematical model to analyze a class of neighbor discovery strategies in ad hoc networks with directional-mode-only antennas. Using the model, we compared different neighbor discovery strategies via numerical analysis and concluded that the a fully synchronized approach where the sector size was equal to the antenna beamwidth performed better than the randomized strategy.

\section{REFERENCES}

[1] X. An and R. Hekmat. Self-adaptive neighbor discovery in ad hoc networks with directional antennas. In Proc. IST Mobilesummit, 2007.

[2] L. Bao and J. Garcia-Luna-Aceves. Transmission scheduling in ad hoc networks with directional antennas. In Proc. ACM MOBICOM, 2002.

[3] E. Gelal, G. Jakllari, S. V. Krishnamurthy, and N. E. Young. An integrated scheme for fully-directional neighbor discovery and topology management in mobile ad hoc networks. In Proc. IEEE MASS, 2006.

[4] G. Pei, M. Albuquerque, J. Kim, D. Nast, and P. Norris. A neighbor discovery protocol for directional antenna networks. In Proc. IEEE MILCOM, 2005.

[5] R. Ramanathan, J. Redi, C. Santivanez, D. Wiggins, and S. Polit. Ad hoc networking with directional antennas: A complete system solution. IEEE J. Select. Areas Commun., 23(3), Mar. 2005.

[6] R. Santosa, B. Lee, C. Yeo, and T. Lim. Distributed neighbor discovery in ad hoc networks using directional antennas. In Proc. IEEE CIT, 2006.

[7] M. Steenstrup. Neighbor discovery among mobile nodes equipped with smart antennas. In Proc. 3rd Scandinavian Workshop on Wireless Ad Hoc Networks, 2003.

[8] H. Takagi and L. Kleinrock. Optimal transmission ranges for randomly distributed packet radio terminals. IEEE Trans. Commun., 32(3), Mar. 1984.

[9] S. Vasudevan, J. Kurose, and D. Towsley. On neighbor discovery in wireless networks with directional antennas. In Proc. IEEE INFOCOM, 2005. 